\title{
25 Research Square \\ Impact of Intradialytic Fiber Clotting on Dialyzer Extraction and Solute Removal: A Randomized Cross-Over Study
}

Floris Vanommeslaeghe

Ghent University Hospital

Iván Josipovic

Ghent University

Matthieu Boone

Ghent University

Wim Van Biesen

Ghent University Hospital

Sunny Eloot ( $\nabla$ sunny.eloot@ugent.be )

Ghent University Hospital

\section{Research Article}

Keywords: Intradialytic Fiber Clotting, Dialyzer Extraction, Solute Removal, biocompatibility, anticoagulation strategy

Posted Date: December 28th, 2021

DOI: https://doi.org/10.21203/rs.3.rs-1128028/v1

License: (c) (i) This work is licensed under a Creative Commons Attribution 4.0 International License. Read Full License

Version of Record: A version of this preprint was published at Scientific Reports on April 5th, 2022. See the published version at https://doi.org/10.1038/s41598-022-09696-7. 


\section{Abstract}

Previous studies revealed the importance of biocompatibility, anticoagulation strategy, and dialysis mode and duration on fiber blocking at the end of a hemodialysis session. The present study was set up in ten hemodialysis patients to relate fiber patency to dialyzer extraction and removal of small and middle molecules. With only $1 / 4$ th of the regular anticoagulation dose, and using a Solacea ${ }^{\mathrm{TM}} 19 \mathrm{H}$ and FX800 CorDiax dialyzer, fiber patency was quantified using 3D micro-CT scanning for different dialysis durations (i.e. 60, 120 and $240 \mathrm{~min}$ ). While Solacea ${ }^{\mathrm{TM}}$ showed good performance in all test sessions, fiber blocking in the FX800 CorDiax did not follow a linear process during dialysis, but was rather accelerated near the end of dialysis. Dialyzer extraction ratios were correlated with the percentages of open fibers. While the fiber blocking process affected extraction ratios (i.e. for phosphorus and myoglobin in the FX800 CorDiax), it had only minor impact on the removal of toxins up to at least $12 \mathrm{kDa}$.

\section{Introduction}

During hemodialysis, the use of anticoagulation is standard of care to prevent clotting of the extracorporeal circuit ${ }^{1}$. Anticoagulation needs to be well-balanced to avoid an increased risk for bleeding complications on the one hand, and clotting of the extracorporeal circuit resulting in blood loss for the patient on the other hand.

Previous research of our group using the gold standard of fiber quantification by micro-CT indicated that a substantial number of fibers can become blocked before this is reflected in a change of routinely observed dialysis parameters or in termination of the dialysis session ${ }^{2}$. We also demonstrated that type of dialyzer (more or less biocompatible), dose and type of anticoagulation strategy, and duration of the dialysis session, next to patient factors such as inflammation, impact substantially on the number of patent fibers at the end of the dialysis session ${ }^{3-8}$.

This study aims to answer two major remaining questions: the kinetics of fiber blocking during the dialysis session, and the degree to which unnoticed fiber blocking impacts overall dialyzer performance in terms of extraction ratio and total solute removal.

Dialyzer extraction ratios for the middle molecules myoglobin and lambda and kappa free light chains, as measured at 1 hour after dialysis start, were not found associated with dialyzer fiber patency post dialysis ${ }^{3}$. This absence of association suggests that fiber blocking is a more delayed phenomenon that occurs only later in the dialysis session. If this would be confirmed, the impact on total solute removal would be limited, as most of the capacity for solute removal of the membrane would still be available during most of the dialysis session.

To investigate the potential impact of fiber blocking on solute clearance in a more robust and representative manner, extraction ratios should be measured just before the dialyzer is disconnected from dialysis and further prepared for micro-CT scanning. Applying such a strategy after different durations of 
dialysis time would not only allow to evaluate the kinetics of percentage fiber blocking over the dialysis session, but also to associate this with the impact on solute clearance and removal.

\section{Results}

Relevant demographic and clinical data of the patient population at baseline (age $57.0 \pm 10.2$; all male) are summarized in Table 1. There were no patient dropouts during the experimental period, scheduled dialysis duration and flow settings were maintained according to the protocol in all the test sessions, and no adverse events were recorded.

Table 1

Demographic and clinical data of the patient population at baseline

\begin{tabular}{|c|c|}
\hline Gender (M/F) & $10 \mathrm{M}$ \\
\hline Age (years) & $57.0 \pm 10.2$ \\
\hline $\begin{array}{l}\text { Dry body } \\
\text { weight (kg) }\end{array}$ & $74.7[69.7 ; 89.1]$ \\
\hline $\begin{array}{l}\text { Dialysis } \\
\text { vintage } \\
\text { (months) }\end{array}$ & $23.1[12.5 ; 40.9]$ \\
\hline Renal disease & $\begin{array}{l}\text { Autosomal dominant polycystic kidney disease }(n=3) \text {; IgA nephropathy }(n=2) \text {; } \\
\text { diabetic nephropathy }(n=1) \text {; reflux nephropathy }(n=1) ; \text { focal segmental } \\
\text { glomerulosclerosis }(n=1) \text {; membranous glomerulonephritis }(n=1) \text {; Alport }(n=1)\end{array}$ \\
\hline $\begin{array}{l}\text { Regular } \\
\text { anticoagulation } \\
\text { dose }\end{array}$ & Enoxaparin 40mg $(n=5)$; Tinzaparin $3500(n=4)$; Enoxaparin 60mg $(n=1)$ \\
\hline $\begin{array}{l}\text { Platelet } \\
\text { inhibitors }\end{array}$ & Acetylsalicylic acid $80 \mathrm{mg}(\mathrm{n}=7)$ \\
\hline $\mathrm{Hb}(\mathrm{g} / \mathrm{dL})$ & $12.0 \pm 0.7$ \\
\hline $\begin{array}{l}\text { Platelets count } \\
\left(10^{3} / \mu \mathrm{L}\right)\end{array}$ & $224 \pm 56$ \\
\hline aPTT (s) & $34.6 \pm 2.6$ \\
\hline INR (-) & $1.0 \pm 0.1$ \\
\hline $\mathrm{CRP}(\mathrm{mg} / \mathrm{L})$ & $5.2[2.3 ; 7.0]$ \\
\hline
\end{tabular}

Cross-sections halfway the dialyzer outlet potting for all the six experimental dialysis sessions of each patient are presented in figure 1. The lumens of open fibers are visualized as black dots. The number of open fibers in the three non-used Solacea ${ }^{\text {TM }}$ samples was $12095 \pm 1$ and in the non-used FX800 CorDiax dialyzers $13047 \pm 1$. In Table 2 this number is compared for both types of dialyzers in the six test sessions, 
using three different thresholds to define the surface area of an open fiber: i.e. 50,70 and $90 \%$ of the cross-section of a non-used fiber.

Table 2

Percentage of open fibers in the six test sessions $(n=10)$ for the thresholds of 50,70 and $90 \%$ open fiber area

\begin{tabular}{|c|c|c|c|c|c|c|c|}
\hline $\begin{array}{l}\text { Open fiber } \\
\text { area }\end{array}$ & SOL_60 & SOL_120 & SOL_240 & FX_60 & FX_120 & FX_240 & $\begin{array}{l}\text { Friedman P- } \\
\text { value }\end{array}$ \\
\hline $50 \%$ & $\begin{array}{l}100 \\
{[100 ; 100]}\end{array}$ & $\begin{array}{l}100 \\
{[100 ; 100]}\end{array}$ & $\begin{array}{l}99 \\
{[98 ; 100]}\end{array}$ & $\begin{array}{l}90 \\
{[81 ; 98]^{a}} \\
\beta\end{array}$ & $\begin{array}{l}84 \\
{[69 ; 92]^{a}} \\
\beta\end{array}$ & $\begin{array}{l}32 \\
{[27 ; 43]^{\text {a } \beta}}\end{array}$ & $<0.001$ \\
\hline $70 \%$ & $\begin{array}{l}100 \\
{[99 ; 100]}\end{array}$ & $\begin{array}{l}100 \\
{[99 ; 100]}\end{array}$ & $\begin{array}{l}99 \\
{[97 ; 99]}\end{array}$ & $\begin{array}{l}90 \\
{[81 ; 98]^{a}} \\
\beta\end{array}$ & $\begin{array}{l}83 \\
{[68 ; 92]^{a}}\end{array}$ & $\begin{array}{l}31 \\
{[26 ; 41] \text { a } \beta} \\
Y\end{array}$ & $<0.001$ \\
\hline $90 \%$ & $\begin{array}{l}74 \\
{[70 ; 79]}\end{array}$ & $\begin{array}{l}74 \\
{[67 ; 88]}\end{array}$ & $\begin{array}{l}64 \\
{[59 ; 69]}\end{array}$ & $\begin{array}{l}63 \\
{[56 ; 65]}\end{array}$ & $\begin{array}{l}52 \\
{[38 ; 59]^{a}} \\
\beta\end{array}$ & $\begin{array}{l}14[9 ; 17] \\
a \beta y\end{array}$ & $<0.001$ \\
\hline \multicolumn{8}{|c|}{$\begin{array}{l}\text { median [25pct;75pct]; SOL: Solacea; FX: FX800 CorDiax; _60: dialysis of 60min; _120: dialysis of } \\
\text { 120min;_240: dialysis of 240min }\end{array}$} \\
\hline${ }^{a} P<0.05 v$ & Is SOL_ & $P<0.05 v$ & $500 L_{-} 12$ & 0 & - & & \\
\hline
\end{tabular}

Extraction ratios (ERs) in the Solacea ${ }^{\mathrm{TM}}$ and $\mathrm{FX} 800$ CorDiax for phosphorus, $\beta 2 \mathrm{M}$, and myoglobin are presented in Table 3 and figure 2 for the different dialysis durations. End dialysis ERs for the investigated solutes are equal for the three different dialysis durations in the Solacea ${ }^{\mathrm{TM}}$ dialyzer, while in the FX800 CorDiax they were substantially lower at $240 \mathrm{~min}$ versus $60 \mathrm{~min}$ dialysis. Furthermore, phosphorus and myoglobin ERs at 240min dialysis in the FX800 CorDiax were also lower than the ERs in the Solacea ${ }^{\mathrm{TM}}$ at any measured time point. 
Table 3

Extraction ratios for phosphorus, $\beta_{2} M$ and Myoglobin in the six dialysis test sessions $(n=10)$

\begin{tabular}{|c|c|c|c|c|c|c|c|}
\hline ER (\%) & SOL_60 & SOL_120 & SOL_240 & FX_60 & FX_120 & FX 240 & Anova $\mathrm{P}$ \\
\hline Phosphorus & $87 \pm 2$ & $86 \pm 4$ & $84 \pm 2$ & $88 \pm 3$ & $85 \pm 3$ & $\begin{array}{l}66 \pm \\
12^{\mathrm{a} \beta \gamma \delta \varepsilon}\end{array}$ & $<0.001$ \\
\hline$\beta_{2} \mathrm{M}$ & $45 \pm 5$ & $38 \pm 7$ & $42 \pm 8$ & $51 \pm 13$ & $50 \pm 5$ & $30 \pm 17^{a \delta \varepsilon}$ & $<0.001$ \\
\hline Myoglobin & $37 \pm 4$ & $36 \pm 5$ & $36 \pm 5$ & $\begin{array}{l}25 \pm \\
11^{\mathrm{a} \beta \gamma}\end{array}$ & $\begin{array}{l}20 \pm \\
4^{\mathrm{a} \gamma \gamma}\end{array}$ & $13 \pm 7^{\mathrm{a} \beta \gamma \delta}$ & $<0.001$ \\
\hline \multicolumn{8}{|c|}{$\begin{array}{l}\beta_{2} \text { M: beta-2-microglobulin; SOL: Solacea; FX: FX800 CorDiax; _60: dialysis of } 60 \mathrm{~min} ;-120 \text { : dialysis of } \\
\text { 120min; _240: dialysis of 240min. }\end{array}$} \\
\hline \multicolumn{8}{|c|}{$\begin{array}{l}{ }^{a} P<0.05 \text { versus SOL_60; } \beta \text { P } P<0.05 \text { versus SOL_120; }{ }^{\vee} P<0.05 \text { versus SOL_240; }{ }^{\delta} P<0.05 \text { versus FX_60; } \varepsilon \\
P<0.05 \text { versus } F X \_120\end{array}$} \\
\hline
\end{tabular}

The relations between the ER as measured at the end of the dialysis session and the visualized percentage of open fibers are shown in figure 3 for the different percentages of open fiber area $(50,70$ and $90 \%$ ). Due to the good performance of the Solacea ${ }^{\mathrm{TM}}$ dialyzer, there is a lack of variation in \% open fibers and, as a consequence, no correlation can be seen for Solacea ${ }^{\mathrm{TM}}$ alone. Combining the results of all test sessions with Solacea ${ }^{\mathrm{TM}}$ and FX800, correlations with \% open fibers were found for phosphorus and myoglobin.

The theoretically calculated cumulative Total Solute Removals (TSR) during the course of a dialysis session of $240 \mathrm{~min}$, and this for the three solutes and two dialyzers under study, are shown in figure 4 (panels A-C) and as an extra function of the percentage open fibers in figure 4 (panels D-F).

\section{Discussion}

To our knowledge, this is the first study to directly link fiber blocking with dialyzer performance in terms of extraction and total solute removal. This cross-over study investigated fiber blocking and dialyzer extraction ratios at different dialysis time points in the Solacea ${ }^{T \mathrm{~T}}$ and FX800 CorDiax dialyzers, during post-dilution hemodiafiltration with reduced anticoagulation dose. While confirming the importance of biocompatibility, it was demonstrated that kinetics of fiber blocking during dialysis is not a linear process, but is accelerated during the second half of dialysis. Furthermore, dialyzer ERs for phosphorus and myoglobin were correlated with the percentages of open fibers, and were consequently lower with the FX800 CorDiax versus Solacea ${ }^{\text {TM }}$. Last, the large decrease in fiber blocking in the FX800 CorDiax during the last part of the dialysis sessions had only minor influence on total solute removal of phosphorus and the middle molecule $\beta_{2} \mathrm{M}$, while myoglobin solute removal was hampered.

Fiber patency in the Solacea ${ }^{\mathrm{TM}}$ dialyzer remained optimal until the end of the $240 \mathrm{~min}$ dialysis session for $50 \%$ and even for $70 \%$ open fiber area, while, mainly due to deposition onto the membrane, fiber patency 
was $74 \%$ (at $60 \mathrm{~min}$ ) down to $64 \%$ (at $240 \mathrm{~min}$ ) when only counting the $90 \%$ open fibers. Apart from this thin layer, percentage open fibers in the FX800 CorDiax were already lower after a 60min dialysis, and were, for $70 \%$ open fiber area, further decreased by $66 \%$ (interval $60-240 \mathrm{~min}$ ) and $63 \%$ (interval 120 $240 \mathrm{~min}$ ). These data confirm earlier work, in which it was demonstrated that better biocompatibility resulted in less activation of the coagulation cascade, even at very low levels of anticoagulation $3,5,9$.

We also observed that fiber blocking is not a smooth process with linear kinetics over time, but rather follows an exponential pattern, accelerating progressively in the later stages of the dialysis session. Our current study setup unfortunately does not allow a minute-by-minute analysis of the coagulation process, as the gold standard micro-CT analysis requests that the dialysis session should be stopped.

Nevertheless, our data do support a rather limited activation of coagulation in the first $120 \mathrm{~min}$ of the dialysis session, despite very low application of anticoagulant, and a more accelerated activation thereafter. Furthermore, in the more biocompatible dialyzers, even after $240 \mathrm{~min}$, fiber blocking was nearly completely absent even when the cut-off is set at a minimal decrease of fiber diameter. This suggests, or is at least compatible with the hypothesis that coagulation is an on/off phenomenon and that once the cascade is activated, there is a rather rapid evolution to complete fiber blocking. However, to our knowledge, accurate tools to quantify fiber blocking online are currently not available to confirm or contradict this hypothesis.

Although a correlation was found between extraction ratio and percentage open fibers, substantial decrease in fiber patency did not result in a proportional decrease in solute extraction. Furthermore, whereas extraction rate decreased by $25 \%$ for the small solute phosphorus, and even by 41 and $48 \%$ for the middle molecules $\beta_{2} \mathrm{M}$ and myoglobin, mathematical analysis showed relatively modest decreases in total solute removal rates of only 6,7 and $9 \%$ for phosphorus, $\beta_{2} M$ and myoglobin, respectively, during the dialysis period $120-240 \mathrm{~min}$. This can be explained by the fact that the instant absolute solute removal is always a proportion of the solute concentration at the dialyzer inlet, and is thus not only determined by the extraction ratio of the dialyzer. Since inlet concentrations are declining during dialysis according to single or multicompartmental solute kinetics in the patient's body, the absolute solute removal will also decline over time. Because fiber blocking was found to be non-linear and most accelerated during the second part of dialysis, the impact of fiber blocking near the dialysis end has a rather limited impact on overall solute removal. This effect is the smallest for those solutes with the highest reduction ratio during dialysis (i.e. with the largest decline from pre to post dialysis concentration), e.g. small water soluble solutes like urea and creatinine ${ }^{10}$. As a result, evaluation of dialyzer coagulation based on urea removal is not representative for fiber patency. The fact that urea clearance might not be the ideal parameter to evaluate dialyzer clotting, is endorsed by previous $\mathrm{K} \cdot \mathrm{t} / \mathrm{V}_{\text {urea }}$ based studies, which did not find significances among different dialysis strategies, while visual inspection and premature termination of the session did ${ }^{11,12}$.

Phosphorus is known to have complex kinetics in the patient, characterized by mobilization of solute transport from the deeper tissues towards the blood, once plasma levels are decreased ${ }^{13,14}$. This results 
in rather constant or even increased dialyzer inlet concentrations during the second half of dialysis, giving the potential of high solute removal in case extraction would have remained constant. Theoretical calculations accordingly reveal that the hampered extraction in the FX800 Cordiax contributed to a loss in total solute removal of only $6.6 \%$. For the more patent Solacea ${ }^{\mathrm{Tm}}$ dialyzer, this loss in total solute removal is even more negligible, and limited to only $0.9 \%$.

Middle molecules like $\beta_{2} \mathrm{M}$ are known to have retarded transport within the patient, a property that plays a more prominent role in solute removal than dialyzer extraction ratio does ${ }^{15-17}$. This implies that for solutes behaving like $\beta_{2} \mathrm{M}$, absolute solute removal is less sensitive to ad hoc changes in extraction. Accordingly, we can explain why the loss in solute removal remained limited to a theoretically calculated $5.6 \%$ (Solacea $^{\mathrm{TM}}$ ) and 7.6\% (FX800 CorDiax), despite observed large differences in extraction ratio at the end of the dialysis session between the two filters. A further reason why both dialyzers perform in the same range is the cross-over in $\beta_{2} \mathrm{M}$ extraction (figure $2 \mathrm{~B}$ ). The somewhat larger sieving coefficient for $\beta_{2} \mathrm{M}$ in the FX800 CorDiax ( 0.85 versus 0.8 in the Solacea $\left.{ }^{\mathrm{TM}}\right)$ explains the higher extraction during the first half of dialysis, while the higher degree in fiber blocking during the second half decreases the dialyzer extraction.

For the middle molecule myoglobin, calculations suggest a $16.5 \%$ loss in total solute removal in the FX800 CorDiax due to the decreasing ER during dialysis. The overall lower myoglobin removal as compared to removal in Solacea ${ }^{\mathrm{TM}}$ was determined by the lower sieving coefficient ( 0.5 in FX800 CorDiax versus 0.8 in Solacea ${ }^{\mathrm{TM}}$ ) from the start of dialysis on, and the lower extraction ratio towards the end of dialysis due to fiber blocking. The patent fibers in the Solacea ${ }^{\mathrm{TM}}$ make this dialyzer superior for the removal of large middle molecules like myoglobin, with a calculated loss in TSR of only $0.9 \%$.

Our study reveals that in routine clinical practice, many dialysis patients might still receive supratherapeutic doses of anticoagulation for their dialysis session. Indeed, despite giving only a quarter of the regular anticoagulation dose, all sessions could be completed successfully. As bleeding complications are still prevalent in chronic dialysis patients, there is room for further optimization of the anticoagulation regime. However, such an optimization is hampered by the lack of an adequate tool to quantify coagulation online during a dialysis session. Complete blocking of the circuitry as a sign of insufficient anticoagulation is a clear and intrusive event, often leading to a permanent increase in dose of anticoagulation. However, over-anticoagulation is less easily detected and therefore, most likely, dose reductions in case of overdosed anticoagulation occur less frequently.

In order to evaluate fiber blocking kinetics, we had to perform separate dialysis sessions of different duration rather than measuring at different time points in a single session, which could be considered a limitation of the study. However, this is the only way to go when using the very sensitive micro-CT scanning technique, the gold standard to quantify dialyzer fiber blocking ${ }^{2,18}$. In addition, patients were their own controls, and to have the best and similar cross-over starting conditions, group randomization was performed per dialyzer. 
As a strength of our study, we were using the same type of dialysis strategy and the same amount of anticoagulant administered always in an identical way (i.e. single bolus at the dialysis start) for all dialysis sessions. This allowed us to make a direct comparison between the dialyzers and between the sessions of different duration.

In conclusion, this study confirmed that biocompatible dialyzers are more resistant to activation of coagulation. Fiber blocking was found to be a non-linear process which is accelerated during the second part of dialysis. While fiber blocking seemed to have only a minor impact on the removal of toxins up to at least $12 \mathrm{kDa}$, the removal of larger toxins like myoglobin was found hampered by fiber blocking.

\section{Patients And Methods}

\section{Patients}

This single center cross-over study (May-June 2021) included ten stable chronic hemodialysis (HD) patients who had stable dialysis sessions during the last 4 weeks, and were not known having a coagulation disorder, active inflammation or malignancy. Double-needle vascular access was achieved through a native arterio-venous fistula.

Power analysis was based on the relative number of patent fibers in a previously performed cross-over study in 10 patients. Power was at least $98 \%(a=0.05)$ using two different types of dialyzers ${ }^{3}$

The protocol adhered to the Declaration of Helsinki, was approved by the institutional research committee (Ethical Committee - Ghent University Hospital, BC 08511 - B6702020000758 - 11/2020), and was registered in www.clinicaltrials.gov (NCT04746391 - 09/02/2021 - 'Impact of Clotting on Dialyzer Efficiency'). Written informed consent was obtained from all included patients.

\section{Dialysis and anticoagulation}

In the study protocol, each patient was dialyzed with six different regimens at midweek, with three different dialysis durations, i.e. 60, 120, and 240min, and two different types of dialyzers, i.e. the ATA ${ }^{\mathrm{TM}}$ Solacea $^{\text {TM }} 19 \mathrm{H}$ dialyzer (Nipro, Osaka, Japan) and the FX800 CorDiax (Fresenius Medical Care, Bad Homburg, Germany).

Patients received a fixed reduced dose (i.e. a quarter of their regular dose) Low-Molecular-Weight Heparin (i.e. enoxaparin Clexane ${ }^{\circledR} 40 \mathrm{mg}$, Sanofi, Belgium) at the beginning of all dialysis sessions, this to amplify the coagulation process.

All test sessions were performed on a 5008 dialysis machine (Fresenius Medical Care, Bad Homburg, Germany) in post-dilution hemodiafiltration with blood flow $300 \mathrm{~mL} / \mathrm{min}$, dialysate flow $500 \mathrm{~mL} / \mathrm{min}$, and substitution at $25 \%$ of blood flow (i.e. $75 \mathrm{~mL} / \mathrm{min}$ ). Ultrafiltration rates were set according to the patient's interdialytic weight gain and clinical status. 
Group randomization (https://www.randomizer.org/) was performed (by the study coordinator) per dialyzer for the three dialysis durations 60,120 , and 240min (Table 4). Each experimental session was preceded with two wash-in sessions using the same type of dialyzer as in the experimental dialysis at midweek, but always with full regular anticoagulation dose. Each patient served as his/her own control.

Table 4

Test protocol

\begin{tabular}{|llllll|}
\hline 1/4 anticoagulation $(\mathbf{n = 1 0})$ & & & \\
\hline 6 test sessions & & & & \\
\hline Solacea $19 \mathrm{H}$ & & FX800CorDiax & \\
Group randomization & & Group randomization & \\
\hline Post-HDF & Post-HDF & Post-HDF & Post-HDF & Post-HDF & Post-HDF \\
60min & 120min & 240min & 60min & 120min & 240min \\
SOL_60 & SOL_120 & SOL_240 & FX_60 & FX_120 & FX_240 \\
\hline Post-HDF: post dilution hemodiafiltration & & \\
\hline
\end{tabular}

Apart from the study protocol, following the study sessions of only 60 and $120 \mathrm{~min}$, patients were dialyzed with a new dialysis circuit for another 180 and $120 \mathrm{~min}$, respectively, to ensure adequate dialysis.

\section{Blood sampling, laboratory and calculations}

At the end of each of the experimental session, blood was sampled from the dialyzer inlet and outlet blood lines. Blood samples were immediately centrifuged and serum was stored at $-80^{\circ} \mathrm{C}$ until batch analysis. Concentrations of the small water-soluble solute phosphorus (molecular weight 31Da) were determined by routine analysis, while those of the middle molecules beta-2-microglobulin ( $\beta 2 \mathrm{M}-11 \mathrm{kDa})$ and myoglobin $(17 \mathrm{kDa})$ were, respectively, determined by ELISA and nephelometry. Colorimetric bromocresol green assay was used to determine Albumin (Alb) concentrations. All analyses were performed in the Routine Laboratory of the Ghent University Hospital, apart from $\beta 2 \mathrm{M}$ for which analyses were done in the Laboratory of the Nephrology Department (Ghent University Hospital).

Dialyzer Extraction Ratios (ER - \%) were calculated from the inlet and outlet serum concentrations $\left(\mathrm{C}_{\text {inlet }}\right.$ and $\mathrm{C}_{\text {outlet }}$ ), and corrected for hemoconcentration (based on Alb levels) for the middle molecules: 
$\operatorname{ER~}(\%)=\frac{\mathrm{C}_{\text {inlet }}-\mathrm{C}_{\text {outlet }} \cdot\left(\mathrm{Alb}_{\text {inlet }} / \mathrm{Alb}_{\text {outlet }}\right)}{\mathrm{C}_{\text {inlet }}} \cdot 100$

Total Solute Removal (TSR) was calculated as the area under the curve of the calculated concentration in spent dialysate. To compare mass removal among different dialysis sessions with different dialyzers and different start concentrations, a mean inlet serum concentration was used per time point $(60,120$, and $240 \mathrm{~min}$ ) and start concentrations (0min) were derived from extrapolation of the slope of the concentration decrease between time point 60 and 120min. From those theoretical inlet serum concentrations, outlet serum concentrations $\left(\mathrm{C}_{\text {outlet }}\right)$ were derived based on the Extraction Ratio (equation $1)$ at the different time points $(60,120$, and $240 \mathrm{~min})$, and dialysate outlet concentrations $\left(C_{\text {dialysate }}\right)$ were derived from the mass balance in the dialyzer as a function of the inlet concentration $\left(C_{\text {inlet }}\right)$, plasma flow $\left(Q_{\text {plasma }}\right)$ as calculated accounting for the hematocrit, dialysate flow $\left(Q_{\text {dialysate }}\right)$, and ultrafiltration rate $\left(Q_{U F}\right)$ :

$$
\begin{aligned}
& C_{\text {inlet }} \cdot Q_{\text {plasma }}=C_{\text {outlet }} \cdot\left(Q_{\text {plasma }}-Q_{U F}\right)+C_{\text {dialysate }} \cdot\left(Q_{\text {dialysate }}+Q_{U F}\right) \\
& C_{\text {dialysate }}=\frac{C_{\text {inlet }} \cdot Q_{\text {plasma }}-C_{\text {outlet }} \cdot\left(Q_{\text {plasma }}-Q_{U F}\right)}{Q_{\text {dialysate }}+Q_{U F}}
\end{aligned}
$$

\section{Micro-CT scanning and coagulation quantification}

At the end of the study session, a standard rinsing procedure of the hemodialyzer was performed with exact $300 \mathrm{~mL}$ rinsing solution. Next, the hemodialyzer was dried for $12 \mathrm{~h}$ applying continuous mild positive pressure ventilation, simultaneously in blood and dialysate compartment. Dialyzer fiber blocking was visualized in the dialyzer outlet potting using a reference micro-CT scanning technique ${ }^{2,18}$.

Octopus Reconstruction software package as licensed by XRE, a Ghent University spin-off company, is used to reconstruct the raw projection data into 2D visualizations. Non-blocked fibers were counted in the central cross-section of the dialyzer outlet potting, using an open-source platform for biological-image analysis (Image $1.51 \mathrm{H}, \mathrm{NIH}$, Bethesda, USA). Three different thresholds were used to define the surface area of an open fiber: i.e. 50,70 , and $90 \%$ of the cross-section of a non-used fiber. Comparing the number of non-blocked fibers in the tested dialyzer with the total number of fibers as measured in three non-used dialyzer samples, provides an objective estimate of the percentage of fiber blocking.

\section{Statistical analysis}

Statistical analyses were performed using SPSS (version 26, SPSS Inc, Chicago, USA). Continuous variables were summarized as mean \pm SD or median [25th percentile; 75th pct]. To compare different related variables, ANOVA tests were performed with Bonferroni post hoc test (normal distributions) or 
Friedman tests with Wilcoxon post hoc test (no normal distributions). To relate different parameters, Spearman correlations were performed.

\section{Declarations}

\section{Acknowledgements}

The authors are indebted to the dialysis nurses, Melanie Mouton, Joris Van Helleputte, Kelly Rokegem, Sabien Inion, Elsie De Man, Ann Scheir, Kris Ampe and Tamara Leune for their help during the clinical study, and to our laboratory staff Tom Mertens and Maria Van Landschoot for the handling and analysis of the blood samples.

\section{Author contributions}

Substantial contribution to the conception and design of the work (FVO, WVB, SE), data acquisition and post processing (IJ, MB), data analysis (FVO, IJ, SE), data interpretation (FVO, SE), drafting the work (SE), substantial revision of the work (FVO, MB, WVB, SE).

\section{Competing Interest statement}

Authors have no competing interest.

\section{Data availability statement}

All data analyzed during this study are included in this published article.

\section{Funding}

Unrestricted grant from Nipro Corporation (Osaka, Japan)

\section{References}

1. Suranyi, M. \& Chow, J. S. Review: anticoagulation for haemodialysis. Nephrology (Carlton) 15, 386392, doi:10.1111/j.1440-1797.2010.01298.x (2010).

2. Vanommeslaeghe, F. et al. Micro-computed tomography for the quantification of blocked fibers in hemodialyzers. Sci Rep 8, 2677, doi:10.1038/s41598-018-20898-w (2018).

3. Vanommeslaeghe, F. et al. A randomized cross-over study with objective quantification of the performance of an asymmetric triacetate and a polysulfone dialysis membrane using different anticoagulation strategies. Clin Kidney J 14, 398-407, doi:10.1093/ckj/sfz163 (2021). 
4. Sunohara, T. \& Masuda, T. Fundamental Characteristics of the Newly Developed ATA Membrane Dialyzer. Contrib Nephrol 189, 215-221, doi:10.1159/000451044 (2017).

5. Vanommeslaeghe, F. et al. How biocompatible haemodialysers can conquer the need for systemic anticoagulation even in post-dilution haemodiafiltration: a cross-over study. Clin Kidney J 14, 17521759, doi:10.1093/ckj/sfaa219 (2021).

6. Jain, N. et al. Role of Platelets in Chronic Kidney Disease. J Am Soc Nephrol, doi:10.1681/ASN.2020121806 (2021).

7. Wen, Y. et al. Dialysis Filter Life, Anticoagulation, and Inflammation in COVID-19 and Acute Kidney Injury. Kidney360 1, 1426, doi:https://doi.org/10.34067/KID.0004322020 (2020).

8. Horl, W. H. Hemodialysis membranes: interleukins, biocompatibility, and middle molecules. J Am Soc Nephrol 13 Suppl 1, S62-71 (2002).

9. Vandenbosch, I. et al. Strategies for asymmetrical triacetate dialyser heparin-free effective haemodialysis: the SAFE study. Clin Kidney J 14, 1901-1907, doi:10.1093/ckj/sfaa228 (2021).

10. Schneditz, D., Platzer, D. \& Daugirdas, J. T. A diffusion-adjusted regional blood flow model to predict solute kinetics during haemodialysis. Nephrol Dial Transplant 24, 2218-2224, doi:10.1093/ndt/gfp023 (2009).

11. Brunot, V. S., J.E.; Mourad, G.; Klouche, K.; Pernin, V.;. Heparin-free renal replacement therapy for chronic hemodialyzed patients at high risk for bleeding: a comparison of on-line predilution hemodiafiltration with conventional hemodialysis. Hemodial Int 22, 463-473 (2018).

12. Matos, F. J. P., B.; Felix, C.; Carvalho, H.; Ponce, P.; Peralta, R.;. Does subjective assessment of dialyzer appearance reflect dialyzer performance in online hemodiafiltration?. Hemodial Int 24, 61-70 (2020).

13. Agar, B. U., Akonur, A., Lo, Y. C., Cheung, A. K. \& Leypoldt, J. K. Kinetic model of phosphorus mobilization during and after short and conventional hemodialysis. Clin J Am Soc Nephrol 6, 28542860, doi:10.2215/CJN.03860411 (2011).

14. Spalding, E. M., Chamney, P. W. \& Farrington, K. Phosphate kinetics during hemodialysis: Evidence for biphasic regulation. Kidney Int 61, 655-667, doi:10.1046/j.1523-1755.2002.00146.x (2002).

15. Leypoldt, J. K. Kinetics of beta2-microglobulin and phosphate during hemodialysis: effects of treatment frequency and duration. Semin Dial 18, 401-408, doi:10.1111/j.1525-139X.2005.00079.x (2005).

16. Stiller, S., Xu, X. Q., Gruner, N., Vienken, J. \& Mann, H. Validation of a two-pool model for the kinetics of beta2-microglobulin. Int J Artif Organs 25, 411-420, doi:10.1177/039139880202500511 (2002).

17. Ward, R. A., Greene, T., Hartmann, B. \& Samtleben, W. Resistance to intercompartmental mass transfer limits beta2-microglobulin removal by post-dilution hemodiafiltration. Kidney Int 69, 14311437, doi:10.1038/sj.ki.5000048 (2006).

18. Claudel, S. E., Miles, L. A. \& Murea, M. Anticoagulation in hemodialysis: A narrative review. Semin Dial 34, 103-115, doi:10.1111/sdi.12932 (2021). 


\section{Figures}
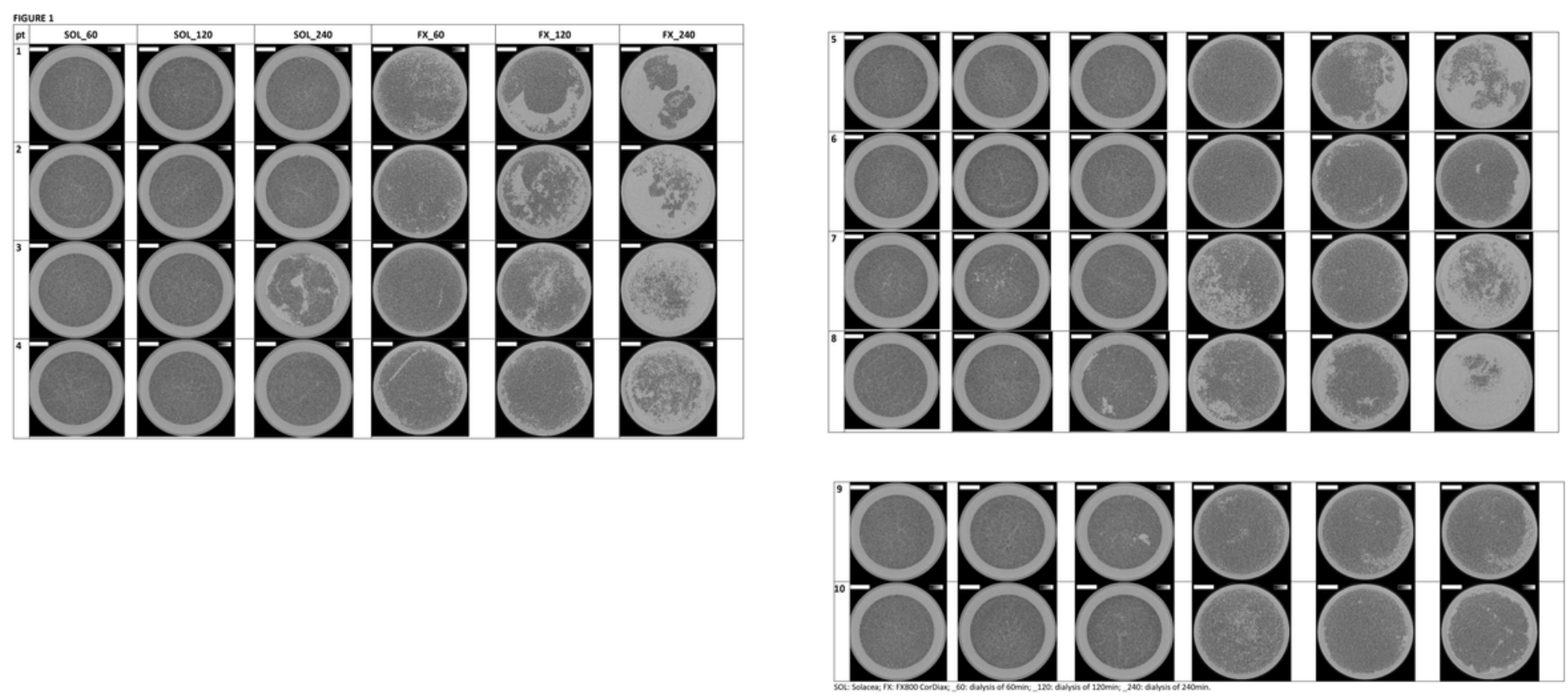

Figure 1

Cross-sections halfway the outlet potting in 10 patients, 2 dialyzers and for each 3 dialysis durations. The greyscale range is from 0 to $0.5 \mathrm{~cm}^{-1}$ and the scale bar denotes $10 \mathrm{~mm}$. 


\section{FIGURE 2}
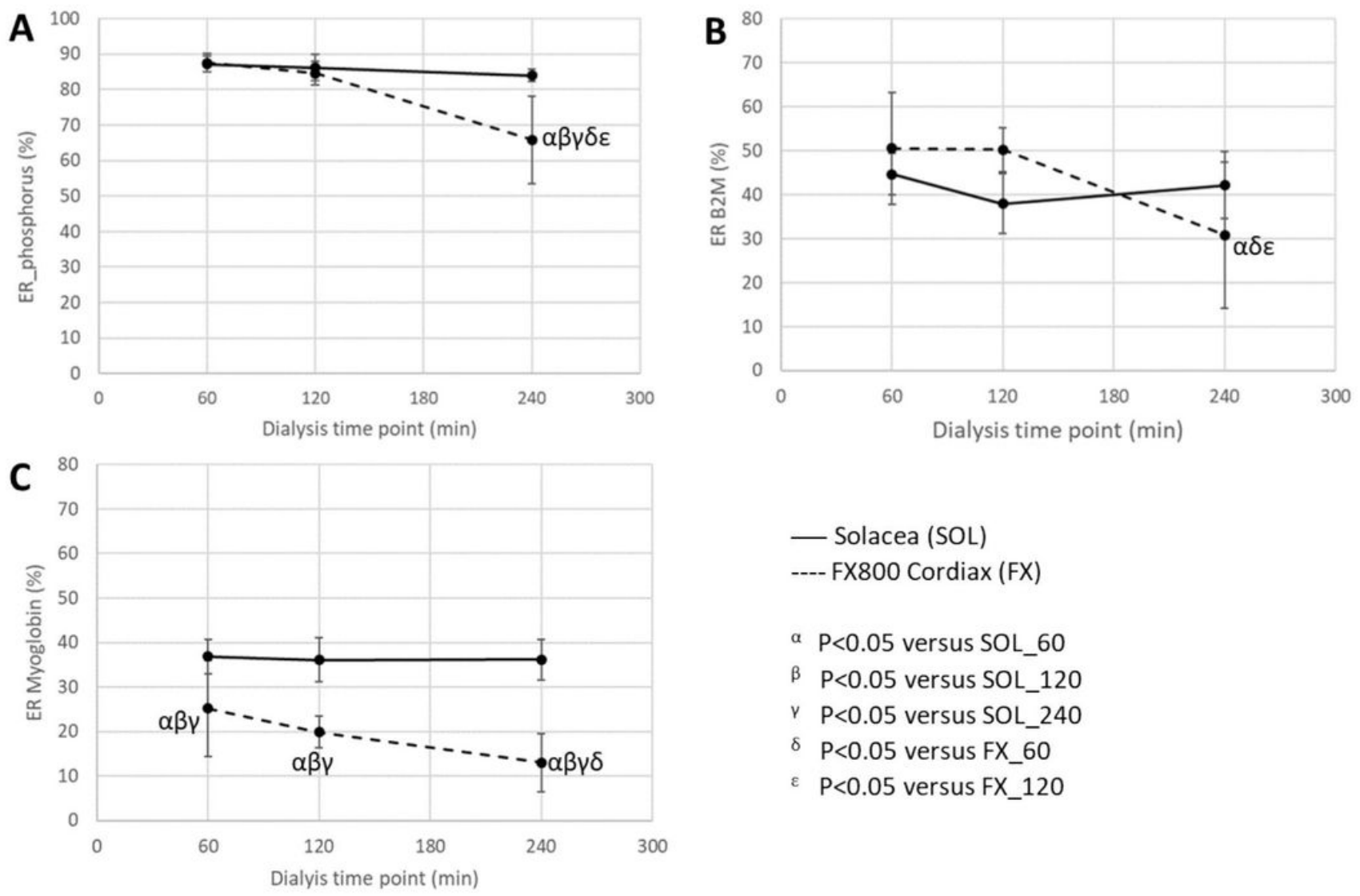

Figure 2

Extraction ratio (ER) of phosphorus (panel A), beta-2-microglobulin (panel B - B2M) and myoglobin (panel C) as measured in 10 patients at the end of a dialysis session with different durations (i.e. 60, 120 and 240min) and with the Solacea (full line) and the FX800 CorDiax dialyzer (dashed line) $(n=10)$. 


\section{FIGURE 3}
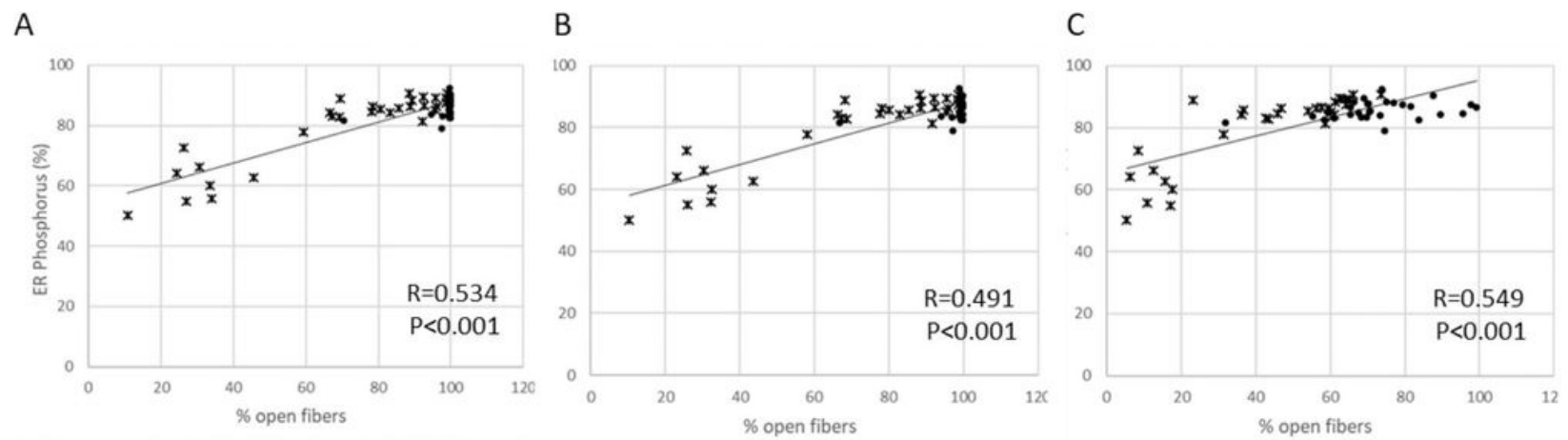

D
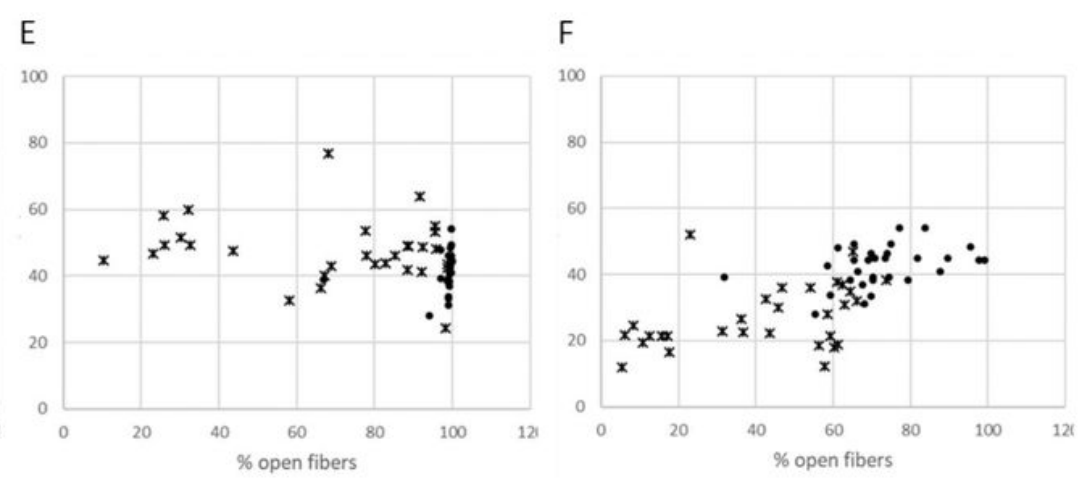

G
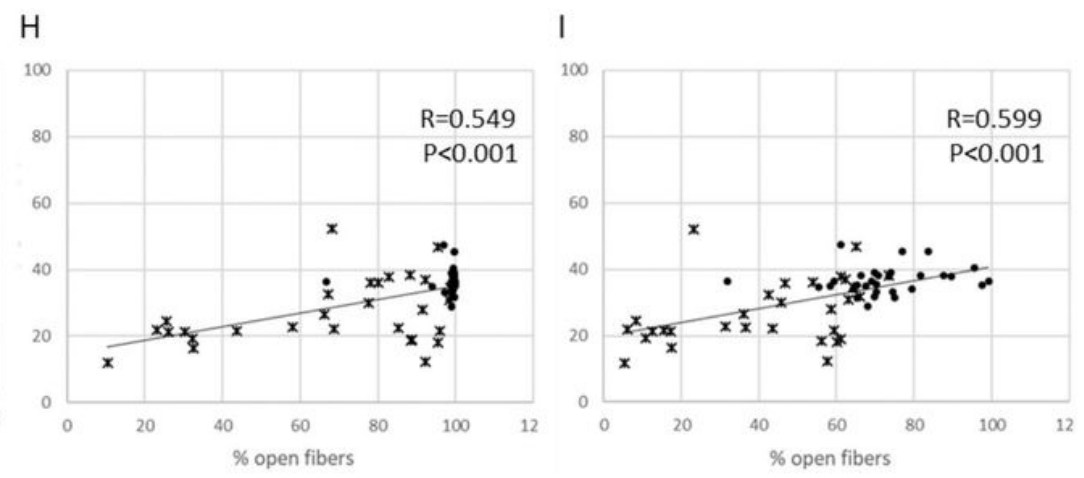

Figure 3

Relations between ER and \% open fibers at the end of dialysis with the Solacea (dots) and the FX800 CorDiax (crosses) for phosphorus (panels A-C), B2M (panels D-F), and Myoglobin (panels G-I) for different \% of open fiber area: 50\% (panels A, D, G), 70\% (Panels B, E, H), and 90\% (panels C, F, I). 

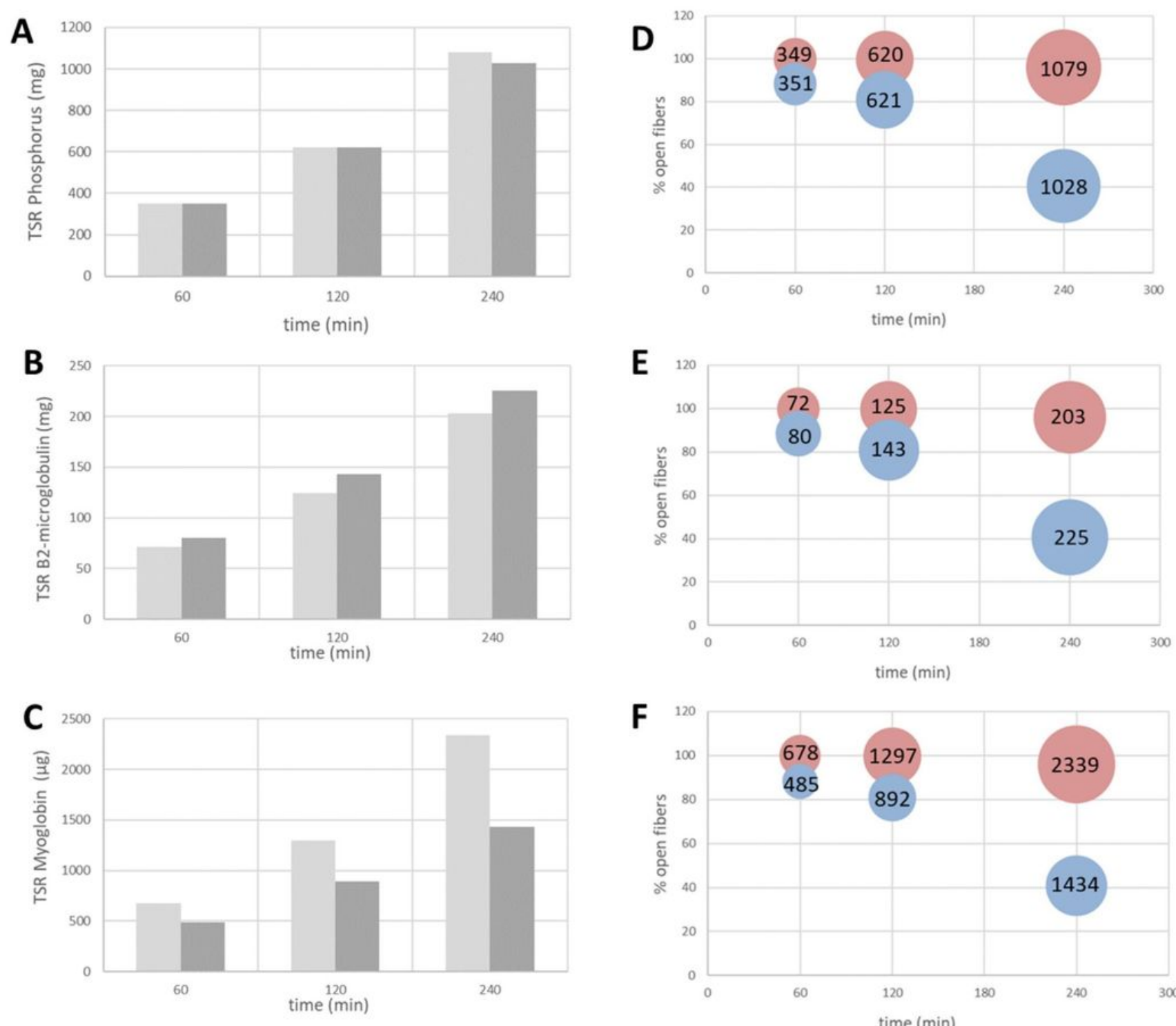

Solacea (SOL)

FX800 Cordiax (FX)

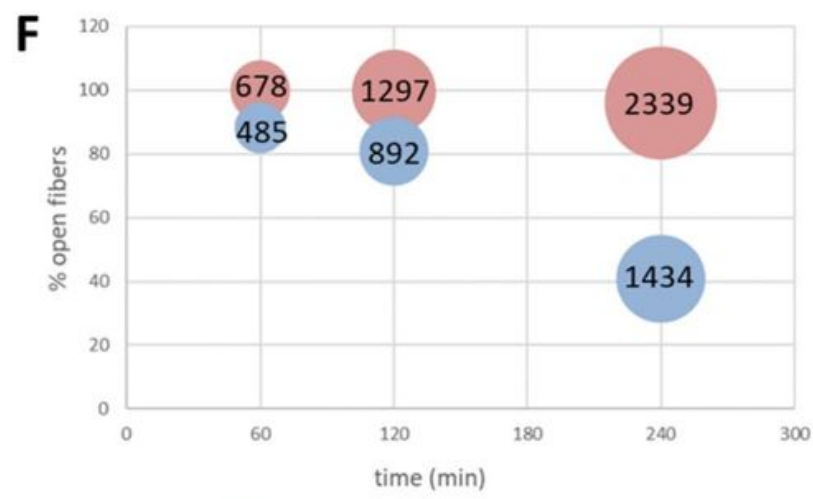

TSR in Solacea (SOL)

TSR in FX800 Cordiax (FX)

\section{Figure 4}

Total Solute Removal as theoretically calculated for a virtual patient, cumulated during dialysis (panels A$C$ ), and as an extra function of the percentage open fibers (panels $D-F$ ) for phosphorus (panel $A+D-m g$ ), $\beta 2$-microglobulin (panel B+E - mg), and Myoglobin (panel $C+F-\mu g$ ). 